\title{
A New Relaxation-Type Sampling Method for Markov Random Image Fields
}

\author{
Masaki Suwa and Sueo Sugimoto \\ Department of Electrical and Electronic Engineering, Ritsumeikan University \\ Noji, Kusatsu City, Shiga 525-77, Japan
}

\begin{abstract}
In this paper, we propose a new sampling method of Markov Random Fields(MRF). Sampling is a kind of generating process for the realization. To obtain such a realization, relaxation-type sampling methods such as Metropolis algorithm or Gibbs sampler are often used. But these sampling algorithms sometimes cause the so called phase transition. Avoiding such a phenomenon, we propose a new Metropolislike sampling algorithm. This algorithm considers the a priori information on the relative frequencies of gray-levels in the original image, and its sampling process can be described by a finite inhomogeneous Markov chain.
\end{abstract}

\section{Introduction}

In this paper, we consider a sampling method on a MRF (or, equivalently, Gibbs random field when cliques on a neighborhood system are introduced).

Markov random field is often applied to texture analysis and synthesis. Especially, in texture synthesis, it is necessary to establish a procedure for generating a texture realization of a MRF image model.

But it is intractable to calculate a joint probability of a texture image based on a Gibbs random field (GRF) because of the existence of the normalizing constant called the partition function in the Gibbs distribution.

Instead of computing the joint probability, the relaxation-type sampling algorithms such as Metropolis algorithm [1],[2] or Gibbs sampler [3] are used. However, these sampling methods sometimes cause the so called phase transition phenomenon [1], which makes the GRF's realization dominated by only few gray-levels. The exchange algorithm [4] which is a sort of Metropolis algorithm can avoid such a phenomenon, but this algorithm does not satisfy the socalled positivity condition of a MRF.

Avoiding the phase transition, we propose a new sampling algorithm based on the Metropolis algorithm. The proposed relaxation-type algorithm contains the a priori information on the relative frequencies of gray-levels in the original image, and its sampling process can be described by a finite inhomogeneous Markov chain. Furthermore, we discuss the stationary distribution of a finite inhomogeneous Markov chain associated with the proposed sampling algorithm. Finally, we perform computer experiments and show that the phase transition can be avoided by applying our proposed sampling method in spite of being relaxation-type.

\section{Gibbs Random Image Model}

We regard the original discrete (finite lattice) image at time $t$ as random variable matrices $\mathbf{X}(t)$ sized $M \times N$ and denote realizations of image as $\{\mathrm{x}\}$. Let $S$ be a set of sites $s$, and let $\eta^{(m)}=\left\{\eta_{s}^{(m)}, s \in S\right\}$ be a $\mathrm{m}$-th order neighborhood system for $S$. We denote $\mathbf{X}=\left\{X_{s}, s \in S\right\}$ as any family of random variables indexed by $S$ and assume a common state space, $\Lambda=\left\{\lambda_{1}, \lambda_{2}, \cdots, \lambda_{K}\right\}$ called "gray-levels". Let $\Omega$ be the set of all possible configurations of image, namely, $\Omega=\left\{\mathrm{x}^{(i)} \mid i=1, \cdots, K^{M N}\right\}$. We can introduce the Gibbs distribution to the image model described by a MRF from the so-called HammersleyClifford Theorem [5]. A Gibbs distribution relative to $\left\{S, \eta^{(m)}\right\}$ is a probability measure $p$ on $\Omega$ with the following representation:

$$
\begin{aligned}
\operatorname{Pr}[\mathbf{X}(t)=\mathbf{x}] & =p(\mathbf{x}) \\
& =\frac{1}{Z(\Theta)} \exp \left(-\frac{U(\mathbf{x} ; \Theta)}{T(t)}\right) \\
Z(\Theta) & =\sum_{\mathbf{x} \in \Omega} \exp \left(-\frac{U(\mathbf{x} ; \Theta)}{T(t)}\right)
\end{aligned}
$$

where $T(t)$ is a function of $t$ that means physical temperature and $\Theta$ is a set of Gibbsian parameters. 
For the present purpose of sampling the GRF, we assume $T(t)=1$. And $U$, called the energy function, determines energy cost of images, is of the form

$$
U(\mathbf{x} ; \Theta) \equiv \sum_{c \in C} V_{c}(\mathbf{x} ; \Theta),
$$

where $C$ denotes the set of cliques, and a family $\left\{V_{c}, c \in C\right\}$ is called a potential.

\section{Sampling from a MRF}

To obtain texture images from the Gibbs random image field model, we have to calculate the Gibbs distribution. But it is always intractable to calculate the probabilities of image $\{\mathrm{x}\}$ due to the normalizing constant $Z(\Theta)$ in (1). Instead, the relaxation-type algorithms are often used such as Metropolis algorithm or Gibbs sampler, which are realized by the finite homogeneous Markov chain. But these sampling algorithms sometimes exhibit a phase transition i.e., the tendency to generate a few gray-level image. Nevertheless this phenomenon is not suitable for texture image modeling, it has not been investigated well in the literature of GRF texture modeling

\subsection{A Metropolis-Like Sampling Method}

To avoid a phase transition, we propose a new sampling algorithm based on Metropolis algorithm. The proposed algorithm can be realized by a kind of finite inhomogeneous Markov chain, which is defined with the following transition probability matrix $\mathbf{P}(t)$. Namely the $(i, j)$ element of $\mathbf{P}$ is given by

$$
\begin{aligned}
{[\mathbf{P}(t)]_{i j} } & =\operatorname{Pr}\left[\mathbf{X}(t+1)=\mathbf{x}^{(j)} \mid \mathbf{X}(t)=\mathbf{x}^{(i)}\right] \\
& \equiv \begin{cases}{[\mathbf{G}(t)]_{i j}[\mathbf{A}(t)]_{i j} ;} & \mathbf{x}^{(j)} \in \Omega_{i} \\
1-\sum_{k \neq i}[\mathbf{P}(t)]_{i k} ; & \mathbf{x}^{(j)}=\mathbf{x}^{(i)} \\
0 ; & \text { otherwise }\end{cases}
\end{aligned}
$$

where $\mathbf{G}(t)$ is called the "generation probability" matrix and $\mathbf{A}(t)$ is called the "acceptance probability" matrix. The $(i, j)$ element of the matrix $\mathbf{G}(t)$ is given by the following definition,

$$
[\mathbf{G}(t)]_{i j} \equiv\left\{\begin{array}{l}
\frac{1}{\alpha^{(j)}} f_{g}\left(a(t) y^{(j)}\right) ; \quad \mathbf{x}^{(j)} \in \Omega_{i} \\
0 ; \quad \text { otherwise, }
\end{array}\right.
$$

$$
y^{(j)} \equiv \pi_{o}\left(x_{\hat{s}}^{(j)}\right)-\pi\left(x_{\hat{s}}^{(j)} ; \mathrm{x}^{(j)}\right)
$$

where $\Omega_{i}$ is the set $\left\{\mathrm{x}^{(j)} \in \Omega\right\}$ which differs from $\mathrm{x}^{(i)}$ in only one site. Clearly, $\mathrm{x}^{(j)} \in \Omega_{i} \Leftrightarrow \mathrm{x}^{(i)} \in$ $\Omega_{j}, \mathrm{x}^{(i)} \notin \Omega_{i} .\left|\Omega_{i}\right|$ is a number of all states $\mathrm{x}$ contained in $\Omega_{i}$. The coefficient $a(t)$ is defined under the following condition;

$$
{ }^{\forall} t>0,{ }^{\exists} B<\infty: \quad 0 \leq a(t) \leq B
$$

A site $\hat{s}$ is chosen randomly or in regular order by raster-scanning, and $\pi\left(x_{\hat{s}}^{(j)} ; \mathrm{x}^{(j)}\right)$ is the relative frequency of the gray-level $x_{\hat{s}}^{(j)}$ for image $\mathrm{x}^{(j)} \cdot \alpha^{(j)}$ is the normalizing constant, and $f_{g}(\cdot)$ is an monotone increasing positive function. Also $\pi_{o}\left(x_{\hat{s}}^{(j)}\right)$ is the relative frequency of the gray-level $x_{\hat{s}}^{(j)}$ for the original image, or an invariant probability of the gray levels $\lambda$ for the GRF model in (1) is adopted if such a probability measure exists and can be evaluated from the GRF model. Namely, we now explain the invariant probability of the gray level: Define the cumulative probability of $x_{s_{i}}$ as follows,

$$
\begin{gathered}
\pi_{o, s_{i}}\left(x_{s_{i}}\right) \equiv \sum_{x_{s_{1}}=\lambda_{1}}^{\lambda_{K}} \cdots \sum_{\left\{\text {except } x_{s_{i}}\right\}} \cdots \\
\times \sum_{x_{s_{M N}}=\lambda_{1}}^{\lambda_{K}} p\left(x_{s_{1}}, x_{s_{2}}, \cdots, x_{s_{M N}}\right) .
\end{gathered}
$$

If a probability $\pi_{o}$ exists such that, for any $s_{i}, \pi_{o}(\lambda)=$ $\pi_{o, s_{i}}(\lambda)$, then $\pi_{o}(\lambda)$ is called an invariant probability of $\lambda$.

The $(i, j)$ element of the acceptance probability matrix $\mathbf{A}$ is also defined by

$$
\equiv\left\{\begin{array}{l}
\min \left[1, \frac{p\left(\mathbf{x}^{(j)}\right) f_{a}\left(a(t) y^{(j)}\right) \alpha^{(j)}}{p\left(\mathrm{x}^{(i)}\right) f_{a}\left(a(t) y^{(i)}\right) \alpha^{(i)}}\right] ; \quad \text { if } \mathrm{x}^{(j)} \in \Omega_{i} \\
0 ; \quad \text { otherwise, }
\end{array}\right.
$$

where $f_{a}(\cdot)$ is defined as a monotone decreasing positive function. We also assume that the following relation holds together with $f_{g}(y)$,

$$
f_{g}(y) \cdot f_{a}(y)=1 .
$$

Here the following question will arise: what is the stationary distribution of the finite inhomogeneous Markov chain with the transition probability in (3)? To reply to this question, we show the following theorem regarding the stationary distribution for the transition probability in (3) with (4) and (5). 


\section{Theorem 1.}

The finite inhomogeneous Markov chain of the transition matrix (3) with (4) and (5) has a stationary distribution which is identical to the one given by the Gibbs distribution in (1).

Theorem 1 is proved in APPENDIX.

\section{Experimental Results}

In this section we show experimental results of synthesizing texture images by the proposed sampling algorithm as well as the Metropolis algorithm from the aspect of observing the phase transition phenomenon. Throughout our experiments, we used a GRF model (the so called first order multilevel logistic (MLL) model [6]) for the nearest neighborhood system $\eta^{(1)}$, with the potential, for $|k|+|l| \leq 1$,

$$
V_{c}\left(x_{(i, j)} \mid \Theta\right)=\left\{\begin{aligned}
-\gamma_{k, l} ; & x_{(i, j)}=x_{(i-k, j-l)} \\
\gamma_{k, l} ; & x_{(i, j)} \neq x_{(i-k, j-l)}
\end{aligned}\right.
$$

where denote $s \equiv(i, j)$ and assume $\gamma_{k, l}=\gamma_{-k,-l}$. The potential parameter of single clique is assumed to be zero. First we generate a $64 \times 64$ original image $(M=N=64)$ with 5 gray-levels, $(\Lambda=$ $\{40,80,120,160,200\})$ by the Metropolis algorithm for the MLL model with potential parameters, $\gamma_{0,1}=$ $0.867, \gamma_{1,0}=1.042$. Beginning initially from an i.i.d. random image, we have the image in Fig. 1 (we call it "IMAGE-1"), after 200 raster scanning $\left(N_{L}=200\right)$. Note that one raster scan contains $M N\left(=64^{2}\right)$ visits from one site to the next site.

Observing the image in Fig. 1, we can not clearly say whether or not the phase transition occurs. Using IMAGE-1 as an original image, we obtain estimates of $\Theta \equiv\left[\gamma_{0,1}, \gamma_{1,0}\right]^{T}$ applying the pseudo likelihood method [7]. These results are listed in Table 1. Next, using these estimated potential parameters in GRF models, we again generated the images by both the Metropolis and the proposed sampling algorithms. Fig. 2 and Fig. 3 show snapshots of the synthesized images at $N_{L}=0,50,100,400,800, \cdots, 2400$, from the top left to the right bottom, by the Metropolis and the proposed sampling algorithms, respectively. In the proposed sampling algorithm, relative frequencies $\pi_{o}(\cdot)$ were determined by counting frequencies of gray levels in IMAGE-1. In Fig. 2 by the Metropolis algorithm, the phase transition phenomenon clearly occurs, i.e. images in Fig. 2 gradu- ally tend to be dominated by one or two gray levels. However such a phenomenon does not occur in textured images in Fig. 3.

Further in our experiments, the functions $f_{g}$ and $f_{a}$ are chosen empirically as follows:

$$
\begin{aligned}
& f_{g}(y)=\exp \{a(t) y\} \\
& f_{a}(y)=\exp \{-a(t) y\}
\end{aligned}
$$

where we set

$$
a(t)=0.1 \cdot \mathbf{1}(t-1000)+1.9 \cdot \mathbf{1}(t-1200),
$$

where $1(\cdot)$ denotes an unit step fuction.

From this experiment, we could confirm that the proposed sampling method can hold visual resemblance as well as the relative frequencies of the graylevel better than the Metropolis algorithm (see TABLE 2).

\section{TABLE 1 Estimated Potential Parameters}

\begin{tabular}{|c|c|c|c|}
\hline & Parameters & True Values & Estimates \\
\hline \multirow{2}{*}{ IMAGE-1 } & $\gamma_{-1,0}$ & 0.867 & 0.890 \\
\cline { 2 - 4 } & $\gamma_{0,-1}$ & 1.042 & 1.029 \\
\hline
\end{tabular}

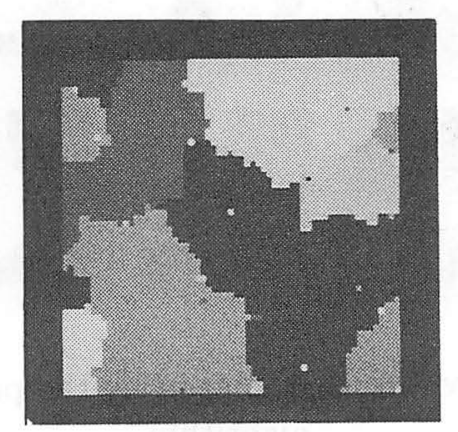

Fig.1 IMAGE-1 


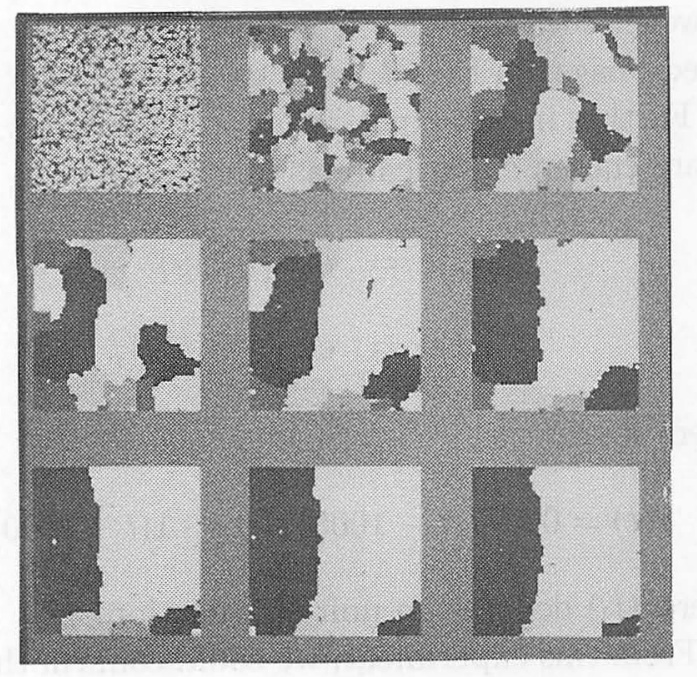

Fig.2 Synthesized imaged by the Metropolis algorithm

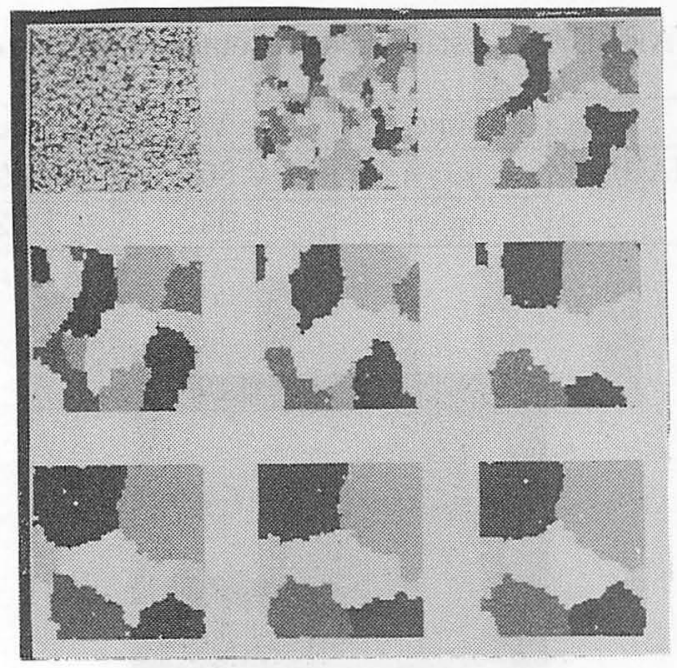

Fig.3 Synthesized imaged by the proposed algorithm

TABLE 2 Relative Frequencies

\begin{tabular}{|c|c|c|c|}
\hline \multirow[b]{2}{*}{ Gray-Levels } & \multicolumn{2}{|c|}{ Sampling Method } & \multirow{2}{*}{$\begin{array}{c}\text { Relative } \\
\text { Frequencies } \\
\text { of IMAGE-1 }\end{array}$} \\
\hline & $\begin{array}{l}\text { Metropolis } \\
\text { Algorithm }\end{array}$ & $\begin{array}{l}\text { Proposed } \\
\text { Algorithm }\end{array}$ & \\
\hline 40 & 0.414 & 0.321 & 0.317 \\
\hline 80 & 0.002 & 0.139 & 0.147 \\
\hline 120 & 0.006 & 0.268 & 0.267 \\
\hline 160 & 0.007 & 0.068 & 0.063 \\
\hline 200 & 0.570 & 0.204 & 0.206 \\
\hline
\end{tabular}

\section{Conclusions}

In this paper, we discussed sampling method generating texture images based on Gibbs random image models. Our proposed Metropolis-like sampling algorithm can avoid a phase transition phenomenon, which containing a priori information on relative frequencies of gray-levels in the original image or on an invariant probability measure of the GRF model. Also it was shown that the stationary distribution of a finite inhomogeneous Markov chain associated with a proposed sampling algorithm is identical to the original Gibbs distribution according to the Markov chain theory. Furthermore, with the computer experiments, we have shown that in comparison to the Metropolis sampling algorithm, our proposed sampling algorithm could avoid the phase transition and simultaneously the feasibility of using this algorithm in texture synthesis.

\section{APPENDIX}

Before we show the proof of Theorem 1, we prepare preliminary results for finite Markov chains, according to [2], [8], [9].

Let $\wp$ be a set of stochastic vector. Assuming that $p_{0} \in \wp$ is an initial stochastic vectors at time $t_{0}(\geq 0)$, we define that $p\left(t_{0}, t ; p_{0}\right)$ is a stochastic vector after transitions from time $t_{0}$ to time $t\left(\geq t_{0}\right)$,

$$
p\left(t_{0}, t ; p_{0}\right) \equiv p_{0} \prod_{l=0}^{l=t-1-t_{0}} \mathbf{P}\left(t_{0}+l\right)
$$

From now on, we represent $\mathbf{Q}\left(t_{0}, t\right) \equiv \prod_{l=0}^{l=t-1-t_{0}} \mathbf{P}\left(t_{0}+\right.$ l). Let $d\left(\mathrm{x}^{(i)}, \mathrm{x}^{(j)}\right)$ be the minimum number of transitions required to change the state $\mathrm{x}^{(i)}$ into the state $\mathrm{x}^{(j)}$.

Furthermore, we define the norm of a vector $p$ as follows;

$$
\|p\|=\sum_{i}\left|[p]_{i}\right| .
$$

\section{Definition 1}

A Markov chain with transition probability matrix $\mathbf{P}(t)$ is weakly ergodic if $\forall t_{0}\left(0 \leq t_{0} \leq t\right)$,

$$
\lim _{t \rightarrow \infty} \sup _{p_{0}, q_{0} \in \wp}\left\{\left\|p\left(t_{0}, t ; p_{0}\right)-p\left(t_{0}, t ; q_{0}\right)\right\|\right\}=0,
$$




\section{Definition 2}

A Markov chain with transition probability matrix $\mathbf{P}(t)$ is strongly ergodic if there exists a stochastic vector $p \in \wp$,

$$
\forall t_{0} \geq 0, \quad \lim _{t \rightarrow \infty} \sup _{p_{0} \in \in_{0}}\left\{\left\|p\left(t_{0}, t ; p_{0}\right)-p\right\|\right\}=0 .
$$

\section{Definition 3}

For $\mathrm{x}^{(i)}, \mathrm{x}^{(j)} \in \Omega$, We define the coefficient of ergodicity $\tau_{1}(\mathbf{P})$ as follows;

$$
\tau_{1}(\mathbf{P}) \equiv 1-\min _{i, j} \sum_{k} \min \left([\mathbf{P}]_{i k},[\mathbf{P}]_{j k}\right) .
$$

\section{Theorem 2}

An inhomogeneous Markov chain with transition probability $\mathbf{P}(t)$ is weakly ergodic if and only if there is a strictly increasing sequence of positive integers $\left\{t_{i} \mid t_{i} \neq t_{i+1}, i=0,1, \cdots\right\}$ such that

$$
\sum_{i=1}^{\infty}\left\{1-\tau_{1}\left(\mathbf{Q}\left(t_{i}, t_{i+1}\right)\right)\right\}=\infty .
$$

\section{Theorem 3}

An inhomogeneous Markov chain with transition probability $\mathbf{P}(t)$ is strongly ergodic if and only if the following conditions are satisfied:

(A) the Markov chain is weakly ergodic,

(B) For all $t \geq 0$, there exists a stochastic vector $p_{t}$ such that $p_{t}=p_{t} \mathbf{P}(t)$,

(C) the stochastic vector $p_{t}$ satisfy

$$
\sum_{t=0}^{\infty}\left\|p_{t}-p_{t+1}\right\|<\infty
$$

Furthermore, if $\lim _{t \rightarrow \infty} p_{t}=p^{*}$, then

$$
\forall t_{0} \geq 0, \quad \lim _{t \rightarrow \infty} \sup _{p_{0} \in \wp}\left\{\left\|p\left(t_{0}, t ; p_{0}\right)-p^{*}\right\|\right\}=0
$$

\section{(Proof of Theorem 1)}

We prove that the transition probability matrix in (3) satisfies conditions (A), (B) and (C) in Theorem 3 and then apply Theorem 3 to prove Theorem 1.

First, we prove that given inhomogeneous Markov chain with transition probability matrix (3) satisfies the condition (A). We define

$$
R \equiv \min _{j}\left\{\max _{i} d\left(\mathrm{x}^{(i)}, \mathrm{x}^{(j)}\right)\right\}
$$

and select the state $\mathrm{x}^{\left(i^{*}\right)}$ such that

$$
\mathrm{x}^{\left(i^{*}\right)} \equiv \arg \min _{j}\left\{\max _{i} d\left(\mathrm{x}^{(i)}, \mathrm{x}^{(j)}\right)\right\} .
$$

Such $R$ and $\mathrm{x}^{\left(i^{*}\right)}$ are certainly exist by assumption (3). Thus for an arbitrary state $\mathrm{x}^{(i)} \in \Omega$, we have the transition probability from $\mathrm{x}^{(i)}$ to $\mathrm{x}^{\left(i^{*}\right)}$, that is,

$$
\begin{aligned}
& {[\mathbf{Q}(t-R, t)]_{i i^{*}}=} \\
& \quad \sum_{i_{1}} \cdots \sum_{i_{R-1}}[\mathbf{P}(t-R)]_{i i_{1}} \cdots[\mathbf{P}(t-1)]_{i_{R-1}, i^{*}}
\end{aligned}
$$

By the definition of $R$, we can extract the state sequence

$$
\begin{array}{ll}
\mathrm{x}^{\left(\hat{i}_{k}\right)} \neq \mathrm{x}^{\left(\hat{i}_{k+1}\right)} & k<m \\
\mathrm{x}^{\left(\hat{i}_{k}\right)}=\mathrm{x}^{\left(i^{*}\right)} & k \geq m
\end{array}
$$

which satisfy

$$
\mathrm{x}^{\left(\hat{i}_{0}\right)}\left(=\mathrm{x}^{(i)}\right), \mathrm{x}^{\left(\hat{i}_{1}\right)}, \cdots, \mathrm{x}^{\left(\hat{i}_{m}\right)},, \cdots, \mathrm{x}^{\left(\hat{i}_{R}\right)}\left(=\mathrm{x}^{\left(i^{*}\right)}\right),
$$

and $0 \leq m \leq R$. Now, we evaluate the transition probability matrix $[\mathbf{P}(t-R+k)]_{\hat{i}_{k}} \hat{i}_{k+1}$ in the following two cases;

(i) $k<m$

By definition (3),

$$
\begin{aligned}
& {[\mathbf{P}(t-R+k)]_{\hat{i}_{k} \hat{i}_{k+1}}} \\
& \quad=[\mathbf{G}(t-R+k)]_{\hat{i}_{k} \hat{i}_{k+1}}[\mathbf{A}(t-R+k)]_{\hat{i}_{k} \hat{i}_{k+1}}
\end{aligned}
$$

and obviously $[\mathbf{G}(t-R+k)]_{\hat{i}_{k} \hat{i}_{k+1}}>0$, we obtain

$$
\begin{aligned}
& {[\mathbf{P}(t-R+k)]_{\hat{i}_{k} \hat{i}_{k+1}}=\frac{f_{g}\left(a(t) y^{\left(\hat{i}_{k+1}\right)}\right)}{\alpha^{\left(\hat{i}_{k+1}\right)}}} \\
& \quad \times \min \left[1, \frac{\exp \left\{-U\left(\mathbf{x}^{\left(\hat{i}_{k+1}\right)}\right)\right\} f_{a}\left(a(t) y^{\left(\hat{i}_{k+1}\right)}\right) \alpha^{\left(\hat{i}_{k+1}\right)}}{\exp \left\{-U\left(\mathrm{x}^{\left(\hat{i}_{k}\right)}\right)\right\} f_{a}\left(a(t) y^{\left(\hat{i}_{k}\right)}\right) \alpha^{\left(\hat{i}_{k}\right)}}\right] \\
& \quad \geq \frac{f_{g}\left(a(t) y^{\left(\hat{i}_{k+1}\right)}\right)}{\alpha^{\left(\hat{i}_{k+1}\right)}} e^{-\Delta U} \frac{f_{a}\left(a(t) y^{\left(\hat{i}_{k+1}\right)}\right) \alpha^{\left(\hat{i}_{k+1}\right)}}{f_{a}\left(a(t) y^{\left(\hat{i}_{k}\right)}\right) \alpha^{\left(\hat{i}_{k}\right)}} \\
& \geq \frac{e^{-\Delta_{U}}}{\alpha^{\left(\hat{i}_{k}\right)}} \frac{1}{f_{a}(-B)},
\end{aligned}
$$

where $\Delta_{U} \equiv \max _{i, j}\left\{U\left(\mathrm{x}^{(j)}\right)-U\left(\mathrm{x}^{(i)}\right)\right\}$

(ii) $k \geq m$ 
By definition of the transition probability matrix we have that

$$
\begin{aligned}
& {[\mathbf{P}(t-R+k)]_{\hat{i}_{k} \hat{i}_{k+1}}=[\mathbf{P}(t-1)]_{\hat{i}_{R} \hat{i}_{R}}} \\
& =1-\sum_{j \neq \hat{i}_{R}}[\mathbf{P}(t-1)]_{\hat{i}_{R} j} \\
& \geq 1-\frac{1}{\alpha^{\left(\hat{i}_{k+1}\right)}} \sum_{j \neq \hat{i}_{R}} f_{g}\left(a(t) y^{(j)}\right)[\mathbf{A}(t)]_{\hat{i}_{R} j} \\
& \geq 1-\frac{1}{\alpha^{\left(\hat{i}_{k+1}\right)}} \sum_{j \neq \hat{i}_{R}} f_{g}\left(a(t) y^{(j)}\right) \\
& \left.\geq 1-\left\{\frac{1}{\alpha^{\left(\hat{i}_{k+1}\right)}} \sum_{j} f_{g}\left(a(t) y^{(j)}\right)-f_{g}\left(a(t) y^{\left(\hat{i}_{R}\right)}\right)\right)\right\} \\
& =f_{g}\left(a(t) y^{\left(\hat{i}_{R}\right)}\right) \geq f_{g}(-B) .
\end{aligned}
$$

From (A6) and (A7) we can obtain the following inequality:

$$
\begin{aligned}
& {[\mathbf{P}(t-R, t)]_{i i_{R}} \geq[\mathbf{P}(t-R)]_{\hat{i}_{0} \hat{i}_{1}} \cdots} \\
& \quad \times[\mathbf{P}(t-R+1)]_{\hat{i}_{m} \hat{i}_{m+1}} \cdots[\mathbf{P}(t-1)]_{\hat{i}_{R-1} \hat{i}_{R}} \\
& \quad \geq\left\{\frac{e^{-\Delta_{U}}}{\alpha^{\left(\hat{i}_{k+1}\right)} f_{a}(-B)}\right\}^{m}\left\{f_{g}(-B)\right\}^{R-m} \\
& \quad=\frac{e^{-m \Delta_{U}}}{\left(\alpha^{\left(\hat{i}_{k+1}\right)}\right)^{m}\left\{f_{a}(-B)\right\}^{R}} .
\end{aligned}
$$

By Definition 3, the coefficient of ergodicity is evaluated as follows,

$$
\begin{aligned}
& \tau_{1}(\mathbf{P}(t-R, t)) \\
& =1-\min _{i, j} \sum_{k} \min \left([\mathbf{P}(t-R, t)]_{i k},[\mathbf{P}(t-R, t)]_{j k}\right) \\
& \leq 1-\min _{i, j} \min \left([\mathbf{P}(t-R, t)]_{i i_{R}},[\mathbf{P}(t-R, t)]_{j i_{R}}\right) \\
& \leq 1-\frac{e^{-m \Delta_{U}}}{\left(\alpha^{\left(\hat{i}_{k+1}\right)}\right)^{m}\left\{f_{a}(-B)\right\}^{R}} .
\end{aligned}
$$

Thus, independently of $t$, we have

$$
1-\tau_{1}(\mathbf{P}(t-R, t)) \geq \frac{e^{-m \Delta_{U}}}{\left(\alpha^{\left(\hat{i}_{k+1}\right)}\right)^{m}\left\{f_{a}(-B)\right\}^{R}} .
$$

Now, we choose an increasing sequence of time $t$,

$$
0, R, 2 R, \cdots, k R, \cdots,
$$

and take the sum of $1-\tau_{1}(\mathbf{P}(t-R, t))$ all over $k$. Hence

$$
\begin{aligned}
\sum_{k=0}^{\infty} 1- & \tau_{1}(\mathbf{P}(k R,(k+1) R)) \\
& \geq \sum_{k=0}^{\infty} \frac{e^{-m \Delta_{U}}}{\left(\alpha^{\left(\hat{i}_{k+1}\right)}\right)^{m}\left\{f_{a}(-B)\right\}^{R}}=\infty .
\end{aligned}
$$

By Theorem 2, an inhomogeneous Markov chain with transition probability matrix given by (2), (3) and (4) is weakly ergodic, which satisfies the condition (A) in Theorem 3.

Next, we show that given Markov chain satisfies condition (B). We use the following lemma to show an existence of the left eigenvector $p_{t}$ of matrix $\mathbf{P}(t)$ for each fixed $t$.

\section{Lemma 1.}

An irreducible Markov chain with transition matrix $\mathbf{P}$ is aperiodic if

$$
\exists \mathrm{x}^{(j)} \in \Omega: \quad[\mathbf{P}]_{j j}>0 .
$$

\section{Lemma 2.}

Let a finite homogeneous Markov chain with transition matrix $\mathbf{P}$ be irreducible and aperiodic. If the elements of a stochastic vector $q$ satisfy the following equation:

$$
[q]_{i}[\mathbf{P}]_{i j}=[q]_{j}[\mathbf{P}]_{j i},
$$

then the stochastic vector $q$ is a stationary distribution.

The relation in (A12) is the so-called detailed balance equation.

First, we show that given a finite homogeneous Markov chain with transition probability matrix $\mathbf{P}$ in (3) with (4) and (5) is irreducible and aperiodic. Irreducibility can be shown as follows:

From the definition of transition probability $\mathbf{P}$ in (3), for any pair $\mathrm{x}^{(i)}$ and $\mathrm{x}^{(j)}$, there exists a finite $n(\geq 1)$, such that

$$
\begin{aligned}
{\left[\mathbf{P}^{n}\right]_{i j} } & =\sum_{j_{1} \in \Omega} \cdots \sum_{j_{n-1} \in \Omega}[\mathbf{P}]_{i j_{1}} \cdots[\mathbf{P}]_{j_{n-1} j} \\
& \geq[\mathbf{G}]_{i k_{1}}[\mathbf{A}]_{i k_{1}} \cdots[\mathbf{G}]_{k_{n-1} j}[\mathbf{A}]_{k_{n-1} j} \\
& >0
\end{aligned}
$$

Namely we can choose states $\mathrm{x}^{\left(k_{1}\right)}, \cdots, \mathrm{x}^{\left(k_{n-1}\right)}$ such that from $\mathrm{x}^{(i)}$ to $\mathrm{x}^{\left(k_{1}\right)}$, from $\mathrm{x}^{\left(k_{1}\right)}$ to $\mathrm{x}^{\left(k_{2}\right)}$, and so on, 
with positive transition probabilities and reaching finally to the state $\mathrm{x}^{(j)}$. Also, aperiodicity is easily shown by Lemma 1 , since $[\mathbf{P}]_{i i}>0$ for any $\mathrm{x}^{(i)}$ from the definition of the acceptance probability in (4)

Next, we show the stochastic vector (1) satisfies the detailed balance equation in Lemma 3 such that the stationary probability exists and coincides with (1) as follows:

Using the definition of the transition probability matrix in (3), we have,

$$
\begin{aligned}
&{ }_{t} \geq 0,{ }_{\mathbf{x}^{(i)} \in} \Omega, \mathrm{x}^{(j)} \in \Omega_{i}: \\
& p\left(\mathrm{x}^{(i)}\right)[\mathbf{P}]_{i j}= p\left(\mathrm{x}^{(i)}\right)[\mathbf{G}(t)]_{i j}[\mathbf{A}(t)]_{i j} \\
&= p\left(\mathrm{x}^{(i)}\right) \frac{f_{g}\left(a(t) y^{(j)}\right)}{\alpha^{(j)}} \\
& \times \min \left(1, \frac{p\left(\mathrm{x}^{(j)}\right) f_{a}\left(a(t) y^{(j)}\right) \alpha^{(j)}}{p\left(\mathrm{x}^{(i)}\right) f_{a}\left(a(t) y^{(i)}\right) \alpha^{(i)}}\right) \\
&= p\left(\mathrm{x}^{(i)}\right) \frac{f_{g}\left(a(t) y^{(j)}\right)}{\alpha^{(j)}} \\
& \times \frac{p\left(\mathrm{x}^{(j)}\right) f_{a}\left(a(t) y^{(j)}\right) \alpha^{(j)}}{p\left(\mathrm{x}^{(i)}\right) f_{a}\left(a(t) y^{(i)}\right) \alpha^{(i)}} \\
& \times \min \left(1, \frac{p\left(\mathrm{x}^{(i)}\right) f_{a}\left(a(t) y^{(j)}\right) \alpha^{(i)}}{p\left(\mathrm{x}^{(i)}\right) f_{a}\left(a(t) y^{(j)}\right) \alpha^{(j)}}\right) \\
&= p\left(\mathrm{x}^{(j)}\right) \frac{f_{g}\left(a(t) y^{(i)}\right)}{\alpha{ }^{(i)}} \\
& \times \min \left(1, \frac{p\left(\mathrm{x}^{(i)}\right) f_{a}\left(a(t) y^{(i)}\right) \alpha^{(i)}}{p\left(\mathrm{x}^{(j)}\right) f_{a}\left(a(t) y^{(j)}\right) \alpha^{(j)}}\right) \\
&= p\left(\mathrm{x}^{(j)}\right)[\mathrm{G}(t)]_{j i}[\mathbf{A}(t)]_{j i} \\
&= p\left(\mathrm{x}^{(j)}\right)[\mathbf{P}(t)]_{j i},
\end{aligned}
$$

where we used the identity: $\min \left(1, \frac{b}{a}\right)=\frac{b}{a} \min \left(1, \frac{a}{b}\right)$ for a positive pair $a$ and $b$, and the relation in (6). Hence by Lemma 3 , the stochastic vector $p$ is a stationary distribution of the Markov chain with transition probability matrix $\mathbf{P}(t)$, namely, the condition (B) in Theorem 3 is satisfied.

Finally, we consider the condition (C).

$$
\sum_{t=0}^{\infty}\left\|p_{t}-p_{t+1}\right\|=0<\infty
$$

because of $p_{t}=p$ (=const.). Therefore, by Theorem 3 , we can conclude that the inhomogeneous Markov chain with transition probability matrix (3), (4)and
(5) is strongly ergodic. Moreover, assume that $t_{0}=$ $0, p^{*}=p$, we obtain

$$
\lim _{t \rightarrow \infty} \sup _{p_{0} \in \wp}\left\{\left\|p\left(0, t ; p_{0}\right)-p\right\|\right\}=0
$$

or

$$
\forall p_{0} \in \wp, \quad \lim _{t \rightarrow \infty} p_{0} \mathbf{Q}(0, t)=p,
$$

which completes the proof of Theorem 1.

\section{References}

[1] R. C. Dubes and A. K. Jain: Random Field Models in Image Analysis, Journal Appl. Stat., Vol 16, No. 2, pp. 131-164 (1989).

[2] D. Mitra: Convergence and Finite-Time Behavior of Simulated Annealing, Adv. Appl. Prob., Vol. 18, pp. 747-771 (1986).

[3] S. Geman and D. Geman: Stochastic Relaxation, Gibbs Distribution and the Bayesian Restoration of Images, IEEE Trans. Pattern Anal. Machine Intell., Vol.PAMI-6, no.6, pp. 721-741 (1984).

[4] G. R. Cross and A. K. Jain: Markov random field texture models, IEEE Trans. Pattern Anal. EMachine Intell., vol. 5, no. 1, pp. 25-39, 1983.

[5] J. Besag: Spatial interaction and the statistical analysis of lattice systems, J. Roy. Statis. Soc. vol. B. 6, no. 2, pp. 192-236, 1974.

[6] H. Derin and W. S. Cole: Segmentation of textured images using Gibbs random fields, Comput. Vision. Graphics \& Image Processing, vol. 35, pp. 72-98, 1986.

[7] J. E. Besag: On the Statistical Analysis of Dirty Pictures, J. Roy. Statis. Soc. B. 48, No. 3, pp. 259-302(1986).

[8] E. Aarts and J. Korst: Simulated Annealing and Boltzmann Machines, John Wiley and Sons, New York (1989).

[9] E. Seneta: Non-negative Matrices and Markov Chains, Springer-Verlag New-York (1981). 
